\title{
A LOW CHARGE STATE ACCELERATION SCHEME FOR POTENTIAL UPGRADE OF THE HRIBF
}

\author{
Y. Zhang*, G. D. Alton \\ Oak Ridge National Laboratory, P.O. Box 2008, Oak Ridge, TN 37831-6368, USA \\ *China Institute of Atomic Energy, Beijing, China
}

\begin{abstract}
This paper describes a low-charge-state heavy-ion post-accelerator system for upgrading the Holifield Radioactive Ion Beam Facility. It is comprised of a room temperature $12.5-\mathrm{MHz} \mathrm{RFQ}$, followed by a normal conducting IH linac, a SC IH linac and a SC QWR linac booster. The system is designed to accelerate ions with mass-to-charge ratio up to 140 . The system can be used to accelerate beams directly (bypass the 25-MV tandem) or to post-accelerate beams from the tandem accelerator. By use of stripping between acceleration stages, low-chargestate radioactive ion beams of all elements, including uranium, can be accelerated to energies above the Coulomb barrier.
\end{abstract}

\section{INTRODUCTION}

This article describes a low-charge-state linac system for potential upgrade of the Holifield Radioactive Ion Beam Facility (HRIBF). A complementary high-chargestate post-acceleration system, based on the use of a highcharge-state ECR ion source, is also described in these proceedings [1]. Although the high-charge-state system is limited to nuclei with mass-to-charge ratio, M/q $\sim 6$, that can be produced in present state-of-the-art high-chargestate ECR ion sources [2], it can efficiently accelerate radioactive nuclei of mass $<150$. However, there are cost advantages as well as drawbacks in choosing a highcharge-state post-accelerator system. For example, ion beams extracted from an ECR ion source are distributed among several charge states, and consequently, the efficiency is lower than can be realized by use of a welldesigned, high-temperature, singly charged ion source. More importantly, ECR ion sources are expensive, and must be shielded from high radiation fields that degrade permanent magnets often used for plasma confinement. To alleviate this problem, radiation insensitive, low-chargestate ion sources are used on-line and their beams injected into the ECR ion source for enhancing the charge state to the value required for acceleration (breeder concept). The breeder concept seriously complicates operation of the facility, increases maintenance and capital costs, and compromises the otherwise respectable efficiency of the ECR ion source for the charge state of interest (typically, $\geq 10 \%$ ), since the overall efficiency is the product of the individual efficiencies of the two sources. (For example, the overall efficiency of a singly charged ion source with $15 \%$ efficiency, injecting into an ECR ion source with $10 \%$ efficiency would have an overall efficiency of $1.5 \%$.) In addition, ECR ion sources are basically cold, thus condensable species can be trapped on the walls and their efficiencies further reduced. Also, in order to preserve optimum efficiency, ECR sources must be operated at pressures more than one order of magnitude lower than high-temperature single-charge ion sources such as the Electron Beam Plasma Ion Source [3].

Even though the low-charge-state linear acceleration scheme is more costly than its high-charge-state counterpart, it is preferred for use at the HRIBF because of the problems cited above and the existence of an inventory of successful low-charge-state ion sources that have been developed for radioactive ion beam generation. The exclusive use of singly charged positive-ion sources in the facility operation on a continuous basis is attractive since these sources are high temperature and highly efficient for most species. The 25-MV tandem is one of principal liabilities of the present HRIBF because of the requirement of injecting negative-ion beams that cannot be formed with high probability for all species. This liability is eliminated by the use of the low-charge-state postacceleration that accelerates positive ions of all species. The system is quite flexible in that beams can be accelerated directly by either bypassing the tandem accelerator or injected into the tandem for postacceleration.. Conceptual design of the light-ion driver accelerator can be found in a technical report [4], and will not be included in this paper.

\section{LOW-CHARGE-STATE SCHEME}

Fig. 1 is a schematic diagram of the low-charge-state RIBs post-accelerator system as a potential upgrade to the present HRIBF. The system is designed to efficiently accelerate singly charged ion beams with masses, $\mathrm{M} \leq$ 140 , for which a number of efficient, high-temperature ion sources have been developed. Ions with $\mathrm{M}>140$ must be doubly charged. These species can be easily generated in simple, high-temperature, ECR ion sources specifically designed to operate in high radiation fields.

To accelerate low-charge-state heavy-ion beams, a $12.5-\mathrm{MHz}$ normal conducting (NC), split-coaxial-type electrode-structure RFQ [5-7], in combination with tanks containing $\mathrm{NC}$ IH cavities, operating at $25 \mathrm{MHz}$, and SC IH cavities operating at 50 and $100 \mathrm{MHz}$, respectively, are chosen as replacements for their high-charge-state counterparts (i.e., the $100-\mathrm{MHz}$ NC RFQ and NC IH cavities described in Ref. 1). The remainder of the lowcharge-state post-accelerator will be identical to the SC QWR linac used in the high-charge-state linac system. 


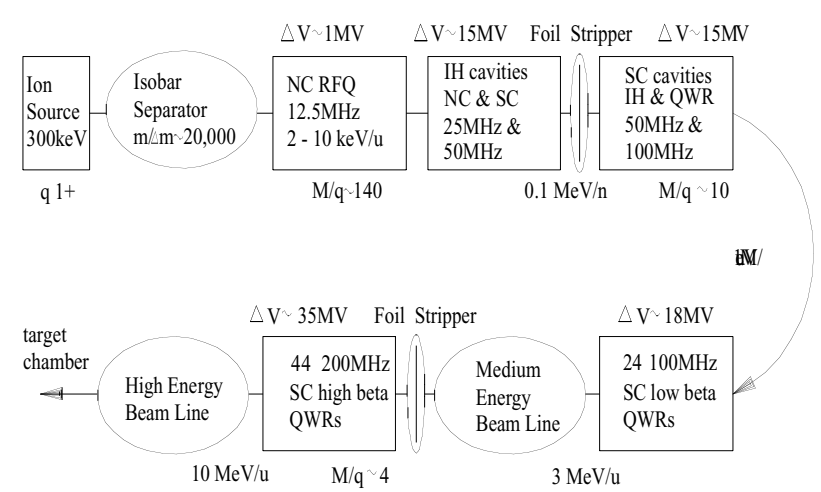

Fig. 1: Schematic diagram of the low-charge-state postaccelerator.

In order to maximize the energy gain for ions with masses $M>140$, it will be necessary to install foil strippers, between the $50-\mathrm{MHz}$ SC IH sections and between the 100- and 200-MHz QWR linac sections, resulting in $\mathrm{M} / \mathrm{q} \cong 10$ and $\mathrm{M} / \mathrm{q} \cong 4$, respectively, as noted in Fig. 1. Under the two-foil stripping scenario, the lowcharge-state system can propel uranium ion beams to energies above $9 \mathrm{MeV} / \mathrm{u}$. However, for heavy ions, the use of the stripping process for the low-charge-state system is analogous to the need for use of a high-chargestate ion source in the high-charge-state scenario described in Ref. 1. The overall efficiency suffers because only $15 \%$ to $30 \%$ of the injected beam is in the most probable charge state. For the two-stripper scenario, the overall efficiency would be reduced to between $\sim 2 \%$ and $\sim 10 \%$. In the case where the energy spread of the ion beam is not a major experimental research issue and the 100- and 200$\mathrm{MHz}$ SC linac sections capture and simultaneously accelerate three to four adjacent charge states, following the respective stripping processes, $\sim 50 \%$ of the injected beam can be accelerated. However, this scenario needs further study.

\section{NC RFQ AND NC IH CAVITIES}

The design parameters of the RFQ are listed in Table 1. Beams with M/q £ 140 can be transported through the device, as simulated with PARMTEQ [8]. In the simulations, the beam transport efficiency of the RFQ is $\sim 94 \%$ where $\sim 81 \%$ of the injected DC beam is captured. The normalized transverse (full) acceptance of the RFQ is $\sim 1 \pi \cdot \mathrm{mm} \cdot \mathrm{mrad}$; the length of the RFQ tank is $\sim 4 \mathrm{~m}$; and the voltage gain is $\sim 1 \mathrm{MV}$. In order to operate at a frequency of $12.5 \mathrm{MHz}$, the split-coaxial RFQ structure has been adopted.

Beams that exit the RFQ must be matched into equivalent frequency IH cavities for efficient capture and acceleration and consequently, very low beta, NC IH cavity structures must be fabricated that meet this velocity matching criterion. In the conceptual design studies, RFQ beams are injected into a $25-\mathrm{MHz}$ normal conducting $\mathrm{IH}$ linac with an overall electrode length of $\sim 3 \mathrm{~m}$, resulting in a voltage gain of $\sim 5 \mathrm{MV}$. Output beams from the
$\mathrm{RFQ} / \mathrm{NC}$ linac combination provide $\beta \cong 0.010$ beams, as required for efficient capture by the following $50-\mathrm{MHz} \mathrm{SC}$ IH linac section.

Table 1. Design parameters for the 12.5-MHz NC RFQ

\begin{tabular}{|l|c|}
\hline Frequency & $12.5 \mathrm{MHz}$ \\
\hline Number of cells & 128 \\
Vane length & $393 \mathrm{~cm}$ \\
Minimum aperture radius & $9.1 \mathrm{~mm}$ \\
Peak vane voltage & $136 \mathrm{kV}$ \\
RF power & $12 \mathrm{~kW}$ \\
Input beam & $1.79 \mathrm{keV} / \mathrm{u}(\beta=0.002)$ \\
Output beam & $9.08 \mathrm{keV} / \mathrm{u}(\beta=0.005)$ \\
\hline
\end{tabular}

\section{SC IH LINAC}

In order to efficiently inject ion beams into the SC linac booster, the 50-MHz and $100-\mathrm{MHz}$ SC IH linac must accelerate beams from $\beta 0.01$ to 0.05 , and thus is designed to do so. The use of SC, rather than NC, IH cavities, is necessitated due to their ability to achieve much higher field gradients and thus, the IH section can be shortened. Although it is attractive to utilize SC structures for all components in the post-accelerator system to reduce RF power consumption, SC structures with optimum beta values $(\beta \leq 0.01)$ are very difficult to construct. For example, SC IH cavities proposed for use at the RIA [9], and the SC RFQs designed at LNL [10], both require $\beta \sim 0.01$ injection velocities. The low-charge-state accelerator will use bulk niobium SC IH structures for the velocity range, $0.01 \leq \beta \leq 0.04$, and bulk niobium SC QWR cavities for the velocity range, $0.04 \leq \beta \leq 0.05$.

The first three cryostats, housing $12,50 \mathrm{MHz}, \mathrm{SC} \mathrm{IH}$ cavities, will accelerate beams from $\beta \sim 0.01$ to of $\beta \sim 0.03$. The first beam stripper will be installed at the entrance to the last $50 \mathrm{MHz}, \mathrm{IH}$ cryostat. The remaining three cryostats, house $4,100 \mathrm{MHz}$ SC IH structures that accelerate beams from $\beta \cong 0.03$ to $\beta \cong 0.04$ and 8,100 $\mathrm{MHz}, \mathrm{SC}$ QWR cavities that accelerate beams with $\mathrm{M} / \mathrm{q}$ 10 to the energies required for injection into the following SC linac booster. The combined voltage gain of the SC IH and SC QWR cavity linac section will be $\sim 25 \mathrm{MV}$. Since bulk niobium SC structures will be used, the average acceleration gradient of the SC IH linac section is expected to be $5 \mathrm{MV} / \mathrm{m}$.

\section{SC LINAC BOOSTER}

State-of-the-art super-conducting cavities have been designed that can accelerate all charged particles, from electrons to the heaviest uranium ions, within the velocity range of $0.05 \leq \beta \leq 1.0$. In recent years, the development of niobium-sputtered, SC cavities for acceleration of heavy ions [11] and electrons [12] has greatly reduced the cost of SC cavity fabrication while exhibiting improved cryogenic RF performance. The SC QWRs will utilize the niobium- sputter coating technology. Independently 
phased QWRs with only two acceleration gaps have broad transit-time-factor curves that make the booster quite flexible for the acceleration of a broad spectrum of RIBs with a wide range of final energies.

Table 2. Design parameters for the SC QWRs

\begin{tabular}{|c|c|c|c|}
\hline & & Low beta & High beta \\
\hline Frequency & $\mathrm{MHz}$ & 100 & 200 \\
\hline$\beta_{\mathrm{opt}}$ & & 0.07 & 0.14 \\
\hline $\mathrm{T}\left(\beta_{\mathrm{opt}}\right)$ & & 0.88 & 0.88 \\
\hline Stored energy/ $\mathrm{E}_{\mathrm{a}}^{2}$ & $\mathrm{~mJ} /(\mathrm{MV} / \mathrm{m})^{2}$ & 95 & 52 \\
\hline $\mathrm{E}_{\mathrm{p}} / \mathrm{E}_{\mathrm{a}}$ & & 5.1 & 5.1 \\
\hline $\mathrm{H}_{\mathrm{p}} / \mathrm{E}_{\mathrm{a}}$ & $\mathrm{G} \cdot \mathrm{m} / \mathrm{MV}$ & 93 & 106 \\
\hline Design $E_{a}$ & $\mathrm{MV} / \mathrm{m}$ & 4.6 & 5.0 \\
\hline
\end{tabular}

The SC linac booster consists of 18 cryostats, the first six house 24 low-beta QWRs followed by 11 cryostats that house 44 high-beta cavities. The last cryostat, houses a QWR that will be used as a debuncher. Quadrupole doublet lenses will be installed between each cryostat to provide transverse focusing. A second beam stripper will be installed between the low- and the highbeta SC QWR sections. The modular aspects of the system allow for construction of the system in stages whenever budgetary restrictions preclude fabrication of the full energy complement. Ions exiting the low-beta section have energies of $\sim 3 \mathrm{MeV} / \mathrm{u}$, sufficiently high for nuclear astrophysics research.

If the first stage is terminated after the low-beta SC QWR section, the energy gain for heavy ions will be only equivalent to that of the present 25-MV tandem, even though the sum of all voltage gains is $\sim 50 \mathrm{MV}$, due to the fact that the charge-states of beams from the linac are lower than those from the tandem. In the second stage, 44 high-beta SC QWRs will be installed that add an additional $35 \mathrm{MV}$ voltage gain so that even uranium beams can be accelerated to energies $\geq 9 \mathrm{MeV} / \mathrm{u}$.

Table 3. Energies of selected low charge-state ion beams

\begin{tabular}{|l|cccccc|}
\hline Ion & $\mathrm{Ni}^{+1}$ & $\mathrm{Kr}^{+1}$ & $\mathrm{Xe}^{+1}$ & $\mathrm{Sm}^{+2}$ & $\mathrm{Au}^{+2}$ & $\mathrm{U}^{+2}$ \\
\hline $\mathrm{E}_{\mathrm{IN}}$ & 0.105 & 0.150 & 0.236 & 0.270 & 0.352 & 0.425 \\
$\mathrm{E}_{\mathrm{RFO}}$ & 0.536 & 0.763 & 1.198 & 1.371 & 1.788 & 2.161 \\
\hline $\mathrm{E}_{1}{ }^{*}$ & 7.9 & 11.3 & 16.8 & 20.3 & 26.5 & 32.0 \\
$\mathrm{Q}_{1}{ }^{*}$ & +9 & +10 & +13 & +14 & +17 & +19 \\
\hline $\mathrm{E}_{2}{ }^{*}$ & 231 & 279 & 390 & 431 & 539 & 621 \\
$\mathrm{Q}_{2}{ }^{*}$ & +22 & +27 & +37 & +41 & +49 & +54 \\
\hline $\mathrm{E}_{\text {OUT }}$ & 993 & 1189 & 1569 & 1710 & 2016 & 2151 \\
$\beta_{\text {OUT }}$ & 0.190 & 0.172 & 0.160 & 0.156 & 0.147 & 0.138 \\
$\mathrm{MeV/u}$ & 16.8 & 14.2 & 11.9 & 11.3 & 10.2 & 9.04 \\
\hline \multicolumn{7}{|c}{ * stripper 1; - stripper 2; energy units: $\mathrm{MeV}$} \\
\hline \multicolumn{7}{|c}{}
\end{tabular}

The low-beta QWRs operate at resonant frequencies of $100 \mathrm{MHz}$ with optimum $\beta \cong 0.07$, while the high-beta QWRs operate at $200 \mathrm{MHz}$ at optimum $\beta \cong 0.14$. Their design parameters are listed in Table 2 . In designing the QWRs, the experiences gained at LNL with niobiumsputtered QWR were adopted. For example, the innerconductor does not incorporate an additional welded or brazed beam tube; the beam hole is bored directly in the cylindrical structure; and the end plate has a curved shape.
Capacitive couplers will be employed in regions of highelectric field to avoid drilling holes in the high current regions of the cavity. Results derived from simulation studies of the transport of several low charge-state ion beams through the post-accelerator system, with the same codes used in Ref. 1 are listed in Table 3.

\section{CONCLUSIONS}

Based on conceptual design studies, the linac postaccelerator system, discussed in this article, will provide a flexible means for accelerating a broad range of radioactive species to research energies. Although the low-charge-state system costs more than its high-chargestate counterpart, described in Ref. 1, due to the additional number of very low-beta SC structures needed, it is preferred for upgrading the HRIBF because of the availability of an inventory of singly charged ion sources and avoidance of the added complexities incumbent with the necessity of using the breeder concept, discussed previously. Since the new linac system can postaccelerate positive-ion beams directly (bypass the tandem) or boost tandem beam energies, RIBs of most elements in the periodic chart will be available for research at the HRIBF.

\section{ACKNOWLEDGEMENTS}

Research sponsored by Oak Ridge National Laboratory, managed by UT-Battelle, LLC, for the U.S. Department of Energy under contract number DE-AC0500OR22725. The authors would like to thank Dr. V. Palmieri and Dr. A. Porcellato of LNL, Dr. S. Takeuchi of JAERI, and Prof. W. Jiang of CIAE for providing information.

\section{REFERENCES}

1. Y. Zhang, G. Alton, Concept Design for a High-Charge-State Acceleration Scheme for Potential Upgrade of the HRIBF, these proceedings, PAC 2001.

2. Z. Q. Xie, Rev. of Sci. Instrum., Vol. 69, No. 2, 1998, p. 625.

3. G. D. Alton, Rev. of Sci. Instrum., Vol. 65, 1994, p. 1141.

4. CONCEPT DESIGN STUDY FOR THE HRIBF DRIVER LINAC - final report to ORNL, ACCSYS Technology Inc., July 1997.

5. J. D. Garrett et al., Proc. $8^{\text {th }}$ Conf. Heavy Ion Accel. Tech., 1998, p. 466.

6. J. A. Nolen et al., Proc. $8^{\text {th }}$ Conf. Heavy Ion Accel. Tech., 1998, p. 477.

7. J. R. J. Bennett $8^{\text {th }}$ Heavy Ion Accel. Tech., 1998, p. 490.

8. K. R. Crandall et al., RFQ DESIGN CODES, LANL, 1998.

9. L. M. Bollinger, LOW- $\beta$ SC LINAC: PAST, PRESENT AND FUTURE, Proc. of LINAC'98.

10. G. Bisoffi et al., First Results With The Full Niobium Superconducting RFQ Resonator At INFN-LNL, Proc. of $7^{\text {th }}$ EPAC, EPAC 2000.

11. A. M. Porcellato et al., Proc. of $8^{\text {th }}$ Int. Conf. on Heavy Ion Accel. Tech., Argonne National Laboratory, Argonne, Illinois, October 1998, p. 228.

12. P. Darriulat, Superconducting Niobium Films for RF Cavities, Proc. of $6^{\text {th }}$ EPAC, EPAC'98, Stockholm, June 1998. 